\title{
On the Design of Intelligent Virtual Instrument for Fault Diagnosis in Mixed Signal Analog Circuit
}

\author{
Ashwani Kumar \\ Electronics \& Communication Engineering Section \\ Yadavindra College of Engineering, Punjabi \\ University, Guru Kashi Campus, Talwandi Sabo.
}

\author{
A.P. Singh \\ Department of Electronics \& Communication \\ Engineering \\ Sant Longowal Institute of Engineering \& \\ Technology, Longowal.
}

\begin{abstract}
Testing of analog and mixed signal circuit has often given bottleneck in the system design. Studies based on the simulation results have been presented by many authors. In this study an intelligent virtual instrument system design for the diagnosis of parametric faults in mixed signal analog circuit is presented. A benchmark R2R digital to analog converter mixed signal circuit is used for fault diagnosis in the study. The impact of circuit component parameter variations on the behavior of a circuit is analyzed by Monte-Carlo analysis. The optimum numbers of test patterns required for fault diagnosis are decided by sensitivity analysis. Finally the fuzzy logic is used for the classification of the faults. Along with fault classification, fuzzy classifier also gives the estimated value of the component faults. The virtual instrument not only displays the diagnosis result but acts as a standalone diagnosis system. The experimental results of the fault diagnosis are presented by both simulated data and the real time data.
\end{abstract}

\section{Index Terms}

Mixed signal circuit, Sensitivity analysis, Monte-Carlo analysis, fuzzy logic, Virtual instrument.

\section{INTRODUCTION}

Fault diagnosis means fault detection and fault isolation. It has the major role in analog and mixed-signal circuit design. Although generally most of the parts of all the electronics system contain digital circuits, but mostly the fault occur in the analog parts of the circuit. The fault diagnosis process in analog and mixed-signal is difficult and complex due to poor fault models, circuit nonlinearities and component tolerances. Due to these reasons the evolution in fault diagnosis in analog and mixed-signal circuits is slow.

The test methods for analog and mixed-signal circuits are classified into two categories, one using the external test equipment and second are the Built-In Self Test (BITS). Both the test methods have been investigated and reported. Fault diagnosis in a digital to analog converter using behavior modeling and hardware description language has been presented [1]. Sensitivity analysis was used at circuit level. In [2] built in self test technique has been presented using ramp generator. In this built in circuit is simulated using a Tspice and $95.22 \%$ fault coverage is reported. Optimum test pattern generator based on GA for the hard fault diagnosis in DAC is also reported [3]. The built in self test [4] also reported for different architectures of digital to analog converters. Parametric fault diagnosis based on wavelet analysis [5] in digital to analog converter claimed $100 \%$ fault diagnosis efficiency. This method represents only simulation results and nothing is reported with real data. As described in [6] the artificial diagnostic techniques can efficiently implemented for the fault diagnosis in analog circuits. So it is clear that intelligent system can be designed for the fault diagnosis in analog and mixed signal circuits. Some of the results [7] for the mixed signal analog circuit using fuzzy math are reported.

In this paper, parametric fault diagnosis in mixed signal analog circuit has been presented. Monte-Carlo simulations of the circuit under test are used to get the behavior the circuit under various parametric variations in the circuit. Test patterns are selected for the diagnosis process using sensitivity analysis. Fault classification is carried out using fuzzy logic. Fault diagnosis results are presented for testing mixed-signal circuit with simulated data and the real time experimental measurement data. Finally a virtual instrument is designed to implement the proposed method. MultiSim, LabVIEW software and the NIELVIS II board are used I this implementation. LabVIEW not only possesses the virtual instruments but also the strong processing capabilities. Due to these capabilities, design of intelligent virtual for the fault diagnosis in a mixed signal analog circuit has become feasible. A benchmark mixed signal R2R digital to analog converter circuit [8] is used to validate the method.

The remaining part of the paper is organized as follows. The section 2 describes the proposed method. The experimental validation of the proposed method both using simulated data and real time data for a benchmark mixed signal circuit are given in section 3. Section 4 describes the results and discussion on the experimental results of the proposed method. Section 5 includes the concluding part of the paper. Acknowledgements and references are given in section 6 and 7 respectively.

\section{PROPOSED METHOD}

The fault diagnosis process in mixed signal circuit can be categorized into three steps: circuit simulation, circuit response measurement and analysis of the measured response. The variation in the circuit responses for faulty and fault free circuits enables the fault diagnosis process. The responses are used as signature parameters for the fault diagnosis process. Faults are classified as catastrophic (Hard) faults and parametric (soft) faults. Catastrophic faults are due to occurrence of open and short circuit in the circuit under test. Parametric faults occur due to the variation in the component values of the circuit. If the parametric value of the component within its nominal range then the circuit is treated as fault free otherwise faulty. In this paper fault diagnosis is implemented only for parametric faults.

\subsection{Sensitivity analysis}

Any change in the circuit response or any other circuit characteristic by a change in its one or more circuit parameters is called as circuit sensitivity. A sensitivity of a 
circuit with response function $y$ with respect to component parameter $x$ is denoted by $S_{x}^{y}$.

It is defined as

$$
S_{x}^{y}=\frac{\lim }{\Delta x \rightarrow 0}\left\{\frac{\frac{\Delta y}{y}}{\frac{\Delta x}{x}}\right\}=\frac{x}{y} \frac{\partial y}{\partial x}
$$

The equation $(i)$ is a general equation for sensitivity and is used for the evaluation of the variation in any circuit parameter, relative to changes in circuit component value. It represents that the percentage that the dependent variable changes, $\frac{\Delta y}{y}$ with respect to the percentage that the independent variable changes, $\frac{\Delta x}{x}$.Taking the limit as the change in $x$ approaches to zero evaluates this ratio for small variations. Sensitivity can be positive or negative. Positive sensitivity means the response function increases as the variation in the component parameter increases and in negative sensitivity response function decreases as the variation in component parameter increases or vice versa.

The analog output of $\mathrm{R} 2 \mathrm{R}$ digital to analog converter is represented as a fraction of the reference $V_{0 u t}=\frac{D_{i}}{2^{N}} V_{\text {ref }}$ where $A_{0}$ is analog output, $\mathrm{D}_{\mathrm{i}}$ is digital input code, $\mathrm{N}$ is number of digital bits and $V_{r e f}$ is the reference voltage. The sensitivity analysis of the DAC determines the effects of the various register parameters affecting the output of the circuit. It has also been seen that each resistance in the R2R network has different sensitivity for each combination of digital input data. It is the total sensitivity of all the resistances which affects the output of the circuit under test.

The fault models are selected with parametric variation $\pm 50 \%$ in the resistance values of the R2R network. To analyze the parametric behavior of the components Monte Carlo analysis of the circuit has been performed. The total sensitivity of all the resistances is calculated for each run of all the defined fault models and fault free condition. The analysis of these sensitivity values is used to derive the number of digital input combinations required to correctly diagnose the faults. Using this technique the input combination which yields in the overlapping of the sensitivity values can be omitted, which in turn simplifies the diagnosis process.

\subsection{Fault classification}

The sensitivity and the Monte Carlo analysis give the minimum number of the input combinations required for the fault diagnosis. These input combinations are applied for each Monte- Carlo run of each defined fault model to get the output voltage of the circuit under test. The nominal value and in each fault model the output voltage values obtained are tabulated and a fault dictionary is constructed. The maximum, minimum and the nominal values of the output response are noted. Fuzzy classifier design has the two major aspects; these include the design of input and output membership functions and the design of fuzzy rule base. The triangular shape of input membership function is depicted by the distribution of output responses of the circuit obtained by Monte Carlo analysis. Triangular membership function is defined by three values $a, b$ and $m$ such that $a<m<b$. The degree of membership is given in equation (iii).

$$
\mu_{t}(x)=\left\{\begin{array}{lr}
0, & x \leq a \\
\frac{x-a}{m-a}, & a<x \leq m \\
\frac{b-x}{b-m}, & m<x<b \\
0, & x \geq b
\end{array}\right.
$$

The shape of the output membership function is depicted as Gaussian shape as it is known that resistance has Gaussian distribution probability function. A Gaussian shape membership function is determined by $\mathrm{c}$ and $\mathrm{k}$. Where $\mathrm{c}$ and $\mathrm{k}$ represents the centre and width of the membership function respectively. The degree membership function is given in equation (iv).

$$
\mu_{g}(x)=e^{-\frac{1}{2}\left(\frac{x-c}{k}\right)^{2}}
$$

\subsection{Virtual instrument design}

Design of the virtual instrument serves two purposes in the present study. In the simulation study of the proposed method it incorporates the fuzzy classifier and displays the fault diagnosis results visually. In real time validation the along with the NI ELVIS II board, virtual instrument acts as a standalone virtual system with fuzzy classification of the faults. It has real time data acquisition, fuzzy classification of faults and displaying of the results. It is designed by using the graphical programming of the LabVIEW. The program designed in LabVIEW consists of front panel and the block diagram. The front panel contains the control variables representing the signatures extracted for the fault diagnosis and the output display units representing the all the components present in the R2R network of mixed-signal digital to analog converter. The block diagram contains the graphical program icons and the interface of the program with the fuzzy system designed for the fault diagnosis.

\section{EXPERIMENTAL VALIDATION}

The proposed method described in the previous section is applied to a benchmark four bit $\mathrm{R} 2 \mathrm{R}$ digital to analog converter mixed signal analog circuit [8]. As this mixed signal analog circuit has already been used previously in other fault diagnosis studies and has been found fairly tolerant to component variations. Hence this circuit is found as a suitable choice for fault diagnosis. Both simulation study and the real time validation results are presented.

\subsection{Simulation study}

The circuit shown in fig. 1 is the benchmark four bit R2R digital to analog converter mixed signal analog circuit. This mixed-signal analog circuit has been taken up to illustrate the proposed method. The output voltage of the circuit under test is given by the equation $(i i)$

$$
V_{0}=\frac{R_{f}}{R} V_{\text {ref }}\left[\frac{D_{0}}{16}+\frac{D_{1}}{8}+\frac{D_{2}}{4}+\frac{D_{3}}{2}\right]
$$

Where $R=10 \mathrm{~K} \Omega$ and hence $2 \mathrm{R}=20 \mathrm{~K} \Omega$. Therefore the values of the resistances in the $\mathrm{R} 2 \mathrm{R}$ network of digital to analog converter are $\mathrm{R} 1=\mathrm{R} 2=\mathrm{R} 3=\mathrm{R} 4=\mathrm{R} 8=20 \mathrm{~K} \Omega$ and $\mathrm{R} 5=\mathrm{R} 6=\mathrm{R} 7=10 \mathrm{~K} \Omega$. Sixteen fault models namely R1 $\uparrow, \mathrm{R} 1 \downarrow$, $\mathrm{R} 2 \uparrow, \mathrm{R} 2 \downarrow, \mathrm{R} 3 \uparrow, \mathrm{R} 3 \downarrow, \mathrm{R} 4 \uparrow, \mathrm{R} 4 \downarrow, \mathrm{R} 5 \uparrow, \mathrm{R} 5 \downarrow, \mathrm{R} 6 \uparrow, \mathrm{R} 6 \downarrow$, $\mathrm{R} 7 \uparrow, \mathrm{R} 7 \downarrow, \mathrm{R} 8 \uparrow$ and $\mathrm{R} 8 \downarrow$ two for each of the resistances $\mathrm{R} 1$ to $\mathrm{R} 8$ are defined. The $\uparrow$ and $\downarrow$ represents the parametric variation in the component value $+50 \%$ and $-50 \%$ respectively. 


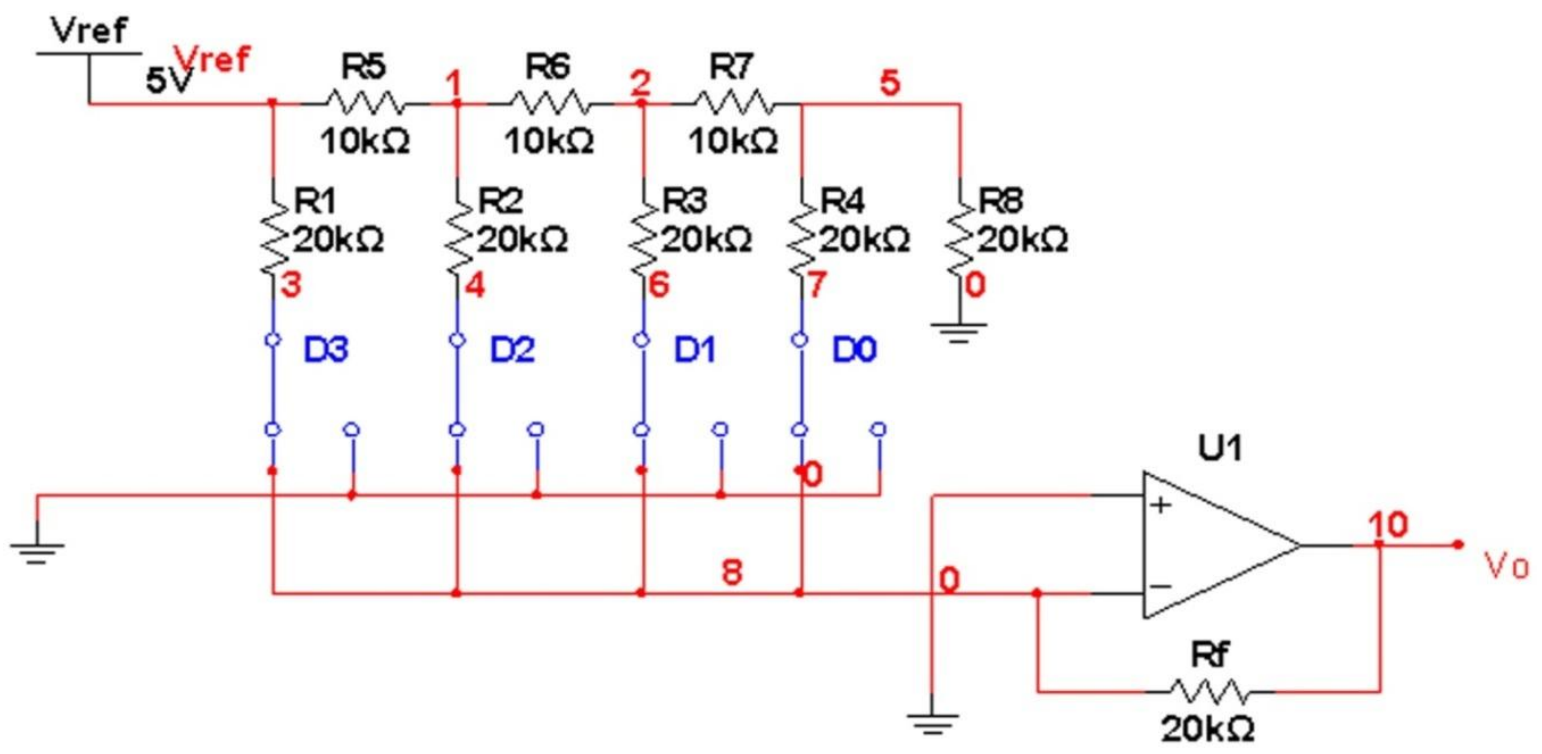

Fig.1 Benchmark R2R digital to analog converter

The output voltage of the DAC is measured for all the sixteen combinations of the input digital signal for all fault and fault free models and the response of the circuit is noted, keeping value of other components to their nominal values. Fifty Monte- Carlo simulations are done for all the fault models and for the fault free condition and the output voltage is measured for each to prepare the fault dictionary. On completing all the simulations for each fault model and fault free model it has been observed that the response value of the circuit is either more or less than the nominal value response. Therefore the maximum output response and the minimum output responses are noted for all fault models. It is needed to mention that for fault free condition, the parametric variation in resistance values remains within the $\pm 5 \%$ of its nominal value.

Now to control the number of test inputs needed for fault diagnosis, the sensitivity analysis of the circuit under test is performed with the help of MULTISIM software for each run of the Monte-Carlo analysis in all the fault models. From sensitivity analysis it has been concluded that the test inputs with input digital value 1 to 8 are sufficient to diagnose the proposed faults. The table 1 shows the fault dictionary with maximum, minimum and the nominal values of the output responses for fault free and faulty models for desired test input signals.

The nominal value is the value of output response when all other components in R2R network of the DAC retain their nominal value for a particular digital input. Maximum and the minimum values are of the output response from fifty Monte Carlo run responses for a given digital input. It is observed that the responses obtained using Monte-Carlo runs oscillate about the nominal value.

\subsubsection{Fuzzy classifier}

The next step in fault diagnosis process is design of fuzzy system for the classification of the faults. Fuzzy system for fault diagnosis is implemented in LabVIEW software by using the fuzzy system designer toolbox. This has been carried out in three steps; these include defining the input membership function, output membership functions and finally designing the rule base.
(a)Defining the input output membership functions This is already mentioned in the previous section that the responses of the circuit obtained for each Monte-Carlo run is either greater than or less than the nominal response. Hence a triangular shape membership function has been depicted and is designed by using minimum, nominal and maximum values of the response output values. Eight inputs are defined for the response of the circuit. Every defined input will have 17 membership functions. Sixteen, one each for the defined fault models and one for the nominal case. In our fuzzy system there are total sixteen single fault models. Hence all inputs in the system has $16 \times 8+8$ (nominal value) $=136$ membership functions. There are eight output variables in the fuzzy classifier, one for each component present in the R2R network of the circuit under test. Each of the output variables contains three membership functions, one for the nominal value and other two for the fault models with parametric variation in the component value $+50 \%$ and $-50 \%$ respectively. The Gaussian shape is selected for the output membership functions as resistors have a Gaussian distribution probability function.

\section{(b)Defining the rules}

Once the membership functions for input and output variables are defined, they have to be connected by IF-THEN rules. The numbers of rules is equal to the number of defined faults. The main advantage of this approach is that it is not required to have the rules for all the cases. The rules only joining the corresponding input output membership function are defined. Since there are 16 fault models and one nominal value, therefore17 IF-THEN rules are designed. The rule structure for the fault $\mathrm{R} 5 \uparrow$ is given as

$$
\begin{aligned}
& \text { If }\{\text { Input1 is } \mathrm{R} 5 \uparrow\} \text { and }\{\text { Input } 2 \text { is } \mathrm{R} 5 \uparrow\} \text { and }\{\text { Input } 3 \text { is } \\
& \mathrm{R} 5 \uparrow\} \ldots \ldots \ldots \text { and }\{\text { Input8 is } \mathrm{R} 5 \uparrow\} \text { then }\{\mathrm{R} 1 \text { is } \mathrm{R} 1\} \text { and } \\
& \{\mathrm{R} 2 \text { is } R 2\} \ldots \ldots \ldots \text { and }\{\mathrm{R} 5 \text { is } \mathrm{R} 5 \uparrow\} \ldots \ldots \ldots \text { and }\{\mathrm{R} 8 \text { is } \mathrm{R} 8\}
\end{aligned}
$$

Where input 1 to input 8 are the responses of the circuits for eight digital inputs and R1, R2, R3, R4, R5, R6, R7 and R8 are the membership function defined for their nominal values within the component tolerance limit of $\pm 5 \%$. The AND (Product) operator is used to combine the antecedents. The main reason to use this operator is that if any of the input signature value falls outside the membership function, the product will be zero and the rule will not be fired. On the 
other hand for min operator the rule be fired and will give the output value according to the computation. Centre of Area (CoA) is used as the defuzzification method in the fuzzy system design. Then after computing all the set of input circuit responses, the fuzzy classifier provide us with estimated value of the components with degree of certainty.

Table: 1 Fault dictionary for the proposed faults

\begin{tabular}{|c|c|c|c|c|c|c|c|c|c|}
\hline $\begin{array}{l}\text { Test } \\
\text { Input }\end{array}$ & & 0001 & 0010 & 0011 & 0100 & 0101 & 0110 & 0111 & 1000 \\
\hline $\begin{array}{l}\text { Fault } \\
\text { Models }\end{array}$ & \multicolumn{9}{|c|}{ Response Output } \\
\hline \multirow{3}{*}{ Nominal } & Max. & -0.5928 & -1.205 & -1.807 & -2.442 & -3.061 & -3.671 & -4.29 & -4.836 \\
\hline & Nominal & -0.6229 & -1.248 & -1.873 & -2.498 & -3.122 & -3.747 & -4.372 & -4.998 \\
\hline & Mini. & -0.6442 & -1.259 & -1.905 & -2.653 & -3.18 & -3.798 & -4.413 & -5.169 \\
\hline \multirow[t]{3}{*}{$\mathrm{R} 1 \uparrow$} & Max. & -0.5928 & -1.205 & -1.807 & -2.442 & -3.061 & -3.671 & -4.29 & -0.331 \\
\hline & Nominal & -0.62192 & -1.2469 & -1.87154 & -2.49682 & -3.12128 & -3.74638 & -4.37114 & -3.33023 \\
\hline & Mini. & -0.6442 & -1.259 & -1.905 & -2.653 & -3.18 & -3.798 & -4.413 & -3.331 \\
\hline \multirow[t]{3}{*}{$\overline{\mathrm{R} 1 \downarrow}$} & Max. & -0.5928 & -1.205 & -1.807 & -2.442 & -3.061 & -3.671 & -4.29 & -9.99555 \\
\hline & Nominal & -0.62192 & -1.2469 & -1.87154 & -2.49682 & -3.12128 & -3.74638 & -4.37114 & -9.99555 \\
\hline & Mini. & -0.6442 & -1.259 & -1.905 & -2.653 & -3.18 & -3.798 & -4.413 & -9.997 \\
\hline \multirow[t]{3}{*}{$\mathrm{R} 2 \uparrow$} & Max. & -0.6539 & -1.319 & -1.978 & -1.623 & -2.457 & -3.124 & -3.783 & -4.836 \\
\hline & Nominal & -0.67874 & -1.36054 & -2.042 & -1.81521 & -2.49645 & -3.17834 & -3.8599 & -4.99655 \\
\hline & Mini. & -0.7006 & -1.364 & -2.059 & -1.837 & -2.535 & -3.2 & -3.898 & -5.169 \\
\hline \multirow[t]{3}{*}{$\mathrm{R} 2 \downarrow$} & Max. & -0.4769 & -0.9613 & -1.442 & -3.941 & -4.423 & -4.907 & -5.388 & -4.836 \\
\hline & Nominal & -0.49692 & -0.99689 & -1.49651 & -3.99636 & -4.4959 & -4.99606 & -5.49589 & -4.99655 \\
\hline & Mini. & -0.5134 & -1.001 & -1.514 & -4.057 & -4.571 & -5.06 & -5.574 & -5.169 \\
\hline \multirow[t]{3}{*}{$\mathrm{R} 3 \uparrow$} & Max. & -0.667 & -0.8938 & -1.563 & -2.496 & -3.185 & -3.423 & -4.112 & -4.836 \\
\hline & Nominal & -0.6946 & -0.92731 & -1.62455 & -2.55497 & -3.25212 & -3.48487 & -4.18225 & -4.99655 \\
\hline & Mini. & -0.7155 & -0.9472 & -1.664 & -2.621 & -3.305 & -3.546 & -4.232 & -5.169 \\
\hline \multirow[t]{3}{*}{$\mathrm{R} 3 \downarrow$} & Max. & -0.4529 & -1.828 & -2.283 & -2.322 & -2.79 & -4.213 & -4.677 & -4.836 \\
\hline & Nominal & -0.47309 & -1.9013 & -2.37727 & -2.37776 & -2.85336 & -4.28185 & -4.75792 & -4.99655 \\
\hline & Mini. & -0.488 & -1.943 & -2.433 & -2.44 & -2.906 & -4.333 & -4.8 & -5.169 \\
\hline \multirow[t]{3}{*}{$\mathrm{R} 4 \uparrow$} & Max. & -0.4422 & -1.238 & -1.696 & -2.456 & -2.921 & -3.721 & -4.186 & -4.836 \\
\hline & Nominal & -0.46493 & -1.28346 & -1.75102 & -2.51144 & -2.97887 & -3.79757 & -4.26523 & -4.99655 \\
\hline & Mini. & -0.4851 & -1.296 & -1.783 & -2.577 & -3.041 & -3.847 & -4.312 & -5.169 \\
\hline \multirow[t]{3}{*}{$\mathrm{R} 4 \downarrow$} & Max. & -0.8943 & -1.131 & -2.044 & -2.413 & -3.346 & -3.569 & -4.503 & -4.836 \\
\hline & Nominal & -0.93776 & -1.17335 & -2.11398 & -2.46741 & -3.40776 & -3.6434 & -4.58423 & -4.99655 \\
\hline & Mini. & -0.9762 & -1.182 & -2.16 & -2.533 & -3.464 & -3.695 & -4.627 & -5.169 \\
\hline \multirow[t]{3}{*}{$\mathrm{R} 5 \uparrow$} & Max. & -0.4788 & -0.9766 & -1.465 & -1.95 & -2.462 & -2.949 & -3.458 & -4.836 \\
\hline & Nominal & -0.49693 & -0.99691 & -1.49657 & -1.99688 & -2.49636 & -2.9965 & -3.4963 & -4.99655 \\
\hline & Mini. & -0.5097 & -1.008 & -1.508 & -2.057 & -2.537 & -3.043 & -3.524 & -5.169 \\
\hline \multirow[t]{3}{*}{$\mathrm{R} 5 \downarrow$} & Max. & -0.8021 & -1.629 & -2.443 & -3.235 & -4.084 & -4.892 & -5.74 & -4.836 \\
\hline & Nominal & -0.83025 & -1.66354 & -2.49648 & -3.33006 & -4.16281 & -4.99618 & -5.82922 & -4.99655 \\
\hline & Mini. & -0.8511 & -1.755 & -2.503 & -3.44 & -4.244 & -5.09 & -5.894 & -5.169 \\
\hline \multirow[t]{3}{*}{$\mathrm{R} 6 \uparrow$} & Max. & -0.5058 & -1.027 & -1.54 & -2.567 & -3.091 & -3.608 & -4.133 & -4.836 \\
\hline & Nominal & -0.5242 & -1.051 & -1.577 & -2.629 & -3.155 & -3.682 & -4.208 & -4.998 \\
\hline & Mini. & -0.5418 & -1.06 & -1.603 & -2.694 & -3.212 & -3.736 & -4.255 & -5.169 \\
\hline \multirow[t]{3}{*}{ R6 $\downarrow$} & Max. & -0.7411 & -1.496 & -2.243 & -2.25 & -3.014 & -3.766 & -4.53 & -4.836 \\
\hline & Nominal & -0.7671 & -1.536 & -2.305 & -2.305 & -3.074 & -3.844 & -4.612 & -4.998 \\
\hline & Mini. & -0.7925 & -1.549 & -2.343 & -2.368 & -3.136 & -3.891 & -4.652 & -5.169 \\
\hline \multirow[t]{3}{*}{$\mathrm{R} 7 \uparrow$} & Max. & -0.5046 & -1.284 & -1.8 & -2.473 & -3.005 & -3.781 & -4.314 & -4.836 \\
\hline & Nominal & -0.5312 & -1.331 & -1.864 & -2.531 & -3.064 & -3.864 & -4.397 & -4.998 \\
\hline & Mini. & -0.549 & -1.347 & -1.895 & -2.596 & -3.122 & -3.91 & -4.437 & -5.169 \\
\hline \multirow[t]{3}{*}{ R7 $\downarrow$} & Max. & -0.716 & -1.089 & -1.817 & -2.394 & -3.145 & -3.504 & -4.255 & -4.836 \\
\hline & Nominal & -0.7525 & -1.13 & -1.884 & -2.451 & -3.205 & -3.582 & -4.337 & -4.998 \\
\hline & Mini. & -0.7788 & -1.145 & -1.919 & -2.514 & -3.269 & -3.632 & -4.376 & -5.169 \\
\hline \multirow[t]{3}{*}{$\mathrm{R} 8 \uparrow$} & Max. & -0.6722 & -1.24 & -1.915 & -2.456 & -3.149 & -3.72 & -4.414 & -4.836 \\
\hline & Nominal & -0.6997 & -1.284 & -1.986 & -2.512 & -3.214 & -3.799 & -4.5 & -4.998 \\
\hline & Mini. & -0.7191 & -1.295 & -2.016 & -2.578 & -3.272 & -3.848 & -4.537 & -5.169 \\
\hline \multirow[t]{3}{*}{ R8\ } & Max. & -0.4487 & -1.132 & -1.582 & -2.412 & -2.876 & -3.568 & -4.032 & -4.836 \\
\hline & Nominal & -0.4684 & -1.174 & -1.644 & -2.468 & -2.938 & -3.644 & -4.115 & -4.998 \\
\hline & Mini. & -0.482 & -1.181 & -1.664 & -2.533 & -2.993 & -3.695 & -4.155 & -5.169 \\
\hline
\end{tabular}




\subsubsection{Virtual instrument design for fault diagnosis} The results of the fault diagnosis i.e. the output of the fuzzy classifier is made available with visual view by a virtual instrument design. It is designed by using the graphical programming of the LabVIEW software. The front panel of the virtual instrument contains eight test input control variables namely response 1 , response $2, \ldots \ldots \ldots$ and response 8

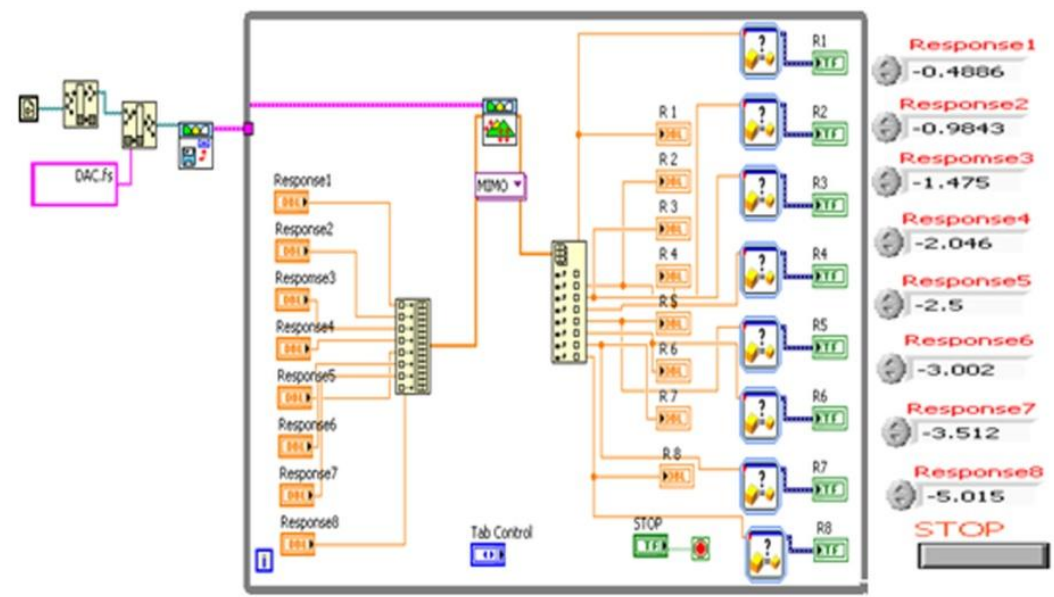

(a) and the display variables R1, R2, R3, R4, R5, R6, R7 and R8. It also includes the estimated value display of the components and the fault indicators. The block diagram of the virtual instrument is designed with the graphical programming. This includes input output blocks, fuzzy classifier and some data processing blocks. The front panel and the block diagram of the virtual instrument are shown in fig.2.

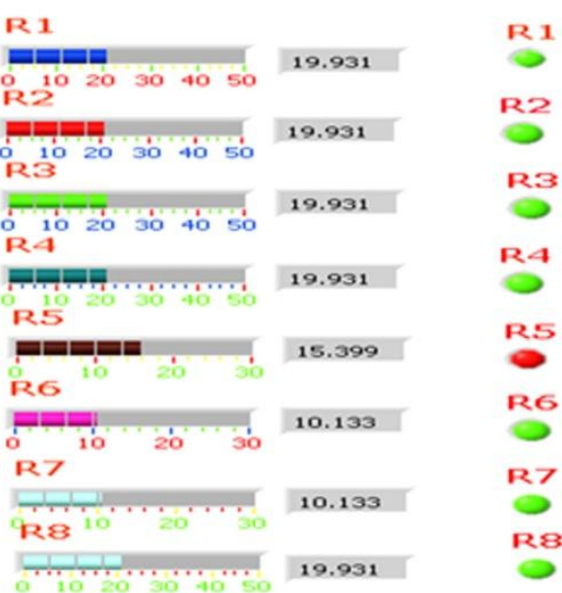

(b)

Fig.2 Proposed Virtual Instrument for fault diagnosis in simulation study (a) Block diagram (b) Front panel

\subsection{Real time validation}

The real time validation is carried out for $\mathrm{R} 2 \mathrm{R}$ digital to analog converter to validate our proposed method using NI ELVIS II board. Similar to the implementations done during the experimental validation of the proposed method using simulation study, same sixteen parametric single faults R1 $\mathrm{R} 1 \downarrow, \mathrm{R} 2 \uparrow, \mathrm{R} 2 \downarrow, \mathrm{R} 3 \uparrow, \mathrm{R} 3 \downarrow, \mathrm{R} 4 \uparrow, \mathrm{R} 4 \downarrow, \mathrm{R} 5 \uparrow, \mathrm{R} 5 \downarrow, \mathrm{R} 6 \uparrow, \mathrm{R} 6 \downarrow$, $\mathrm{R} 7 \uparrow, \mathrm{R} 7 \downarrow, \mathrm{R} 8 \uparrow$ and $\mathrm{R} 8 \downarrow$ are considered. The responses of the circuit under test are measured for these parametric faults with eight digital input signals. The digital to analog converter circuit is implemented using operational amplifier 741 and other required components on the NI ELVIS II board. The fifty response values are obtained for each fault model with all selected number of digital inputs i.e. from 1 to 8 . The table 2 shows one sample of response values obtained for each fault model.

Table: 2 One Sample of real time data values of the output response

\begin{tabular}{|c|c|c|c|c|c|c|c|c|}
\hline Test Input & 0001 & 0010 & 0011 & 0100 & 0101 & 0110 & 0111 & 1000 \\
\hline Fault Models & \multicolumn{8}{|c|}{ Response Output } \\
\hline Nominal & -0.62262 & -1.23888 & -1.8603 & -2.4714 & -3.09217 & -3.70907 & -4.33145 & -4.9029 \\
\hline $\mathrm{R} 1 \uparrow$ & -0.6223 & -1.23824 & -1.85933 & -2.47075 & -3.0912 & -3.70875 & -4.32984 & -3.29006 \\
\hline 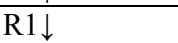 & -0.61781 & -1.23208 & -1.85144 & -2.46097 & -3.07991 & -3.69594 & -4.31623 & -9.59228 \\
\hline $\mathrm{R} 2 \uparrow$ & -0.67446 & -1.34524 & -2.02147 & -1.79955 & -2.47473 & -3.14671 & -3.82367 & -4.89594 \\
\hline R2 $\downarrow$ & -0.49771 & -0.99263 & -1.49161 & -3.91362 & -4.41369 & -4.91163 & -5.41818 & -4.8993 \\
\hline $\mathrm{R} 3 \uparrow$ & -0.69012 & -0.92243 & -1.61369 & -1.61369 & -3.21572 & -3.44864 & -4.14106 & -4.8986 \\
\hline R3 $\downarrow$ & -0.47445 & -1.87322 & -2.35071 & -2.3521 & -2.82753 & -4.22907 & -4.70733 & -4.89957 \\
\hline $\mathrm{R} 4 \uparrow$ & -0.46518 & -1.27103 & -1.73723 & -2.48129 & -2.94714 & -3.75472 & -4.22142 & -4.8974 \\
\hline R4 $\downarrow$ & -0.92806 & -1.16314 & -2.09379 & -2.43918 & -3.36895 & -3.6047 & -4.53709 & -4.89994 \\
\hline$\overline{\mathrm{R} 5 \uparrow}$ & -0.49526 & -0.98718 & -1.48377 & -1.97703 & -2.47339 & -2.96663 & -3.4642 & -4.89925 \\
\hline R5 $\downarrow$ & -0.8242 & -1.64408 & -2.4688 & -3.28814 & -4.11344 & -4.93327 & -5.76296 & -4.9001 \\
\hline R6个 & -0.52178 & -1.04031 & -1.5636 & -2.59814 & -3.12094 & -3.64067 & -4.16477 & -4.90047 \\
\hline R6 $\downarrow$ & -0.76163 & -1.51802 & -2.2816 & -2.27888 & -3.0421 & -3.80025 & -4.56503 & -4.90052 \\
\hline$\overline{\mathrm{R} 7 \uparrow}$ & -0.52782 & -1.31722 & -1.84615 & -2.49931 & -3.02794 & -3.82453 & -4.35489 & -4.90214 \\
\hline $\mathrm{R} 7 \downarrow$ & -0.74585 & -1.11837 & -1.86591 & -2.41967 & -3.16667 & -3.54016 & -4.28907 & -4.90222 \\
\hline $\mathrm{R} 8 \uparrow$ & -0.69606 & -1.27222 & -1.96995 & -2.48202 & -3.17918 & -3.75719 & -4.45603 & -4.89852 \\
\hline R8\ & -0.46503 & -1.16137 & -1.62723 & -2.43721 & -2.9027 & -3.60063 & -4.06707 & -4.89739 \\
\hline
\end{tabular}


The fuzzy classifier is also designed using the fuzzy designer tool box of LabVIEW in the similar way as is designed during simulation study. Triangular shape membership and the Gaussian shape membership functions are used for input and outputs respectively. Finally the virtual instrument is designed. The front panel of the virtual instrument contains display for the eight output responses, digital multimeter tool of NI ELVIS II board, estimated value display of the components and the fault indicators. The block diagram of the virtual instrument contains the blocks of front panel, fuzzy classifier and some data processing blocks. The proposed virtual instrument used for fault diagnosis in real time validation is shown in fig. 3 .

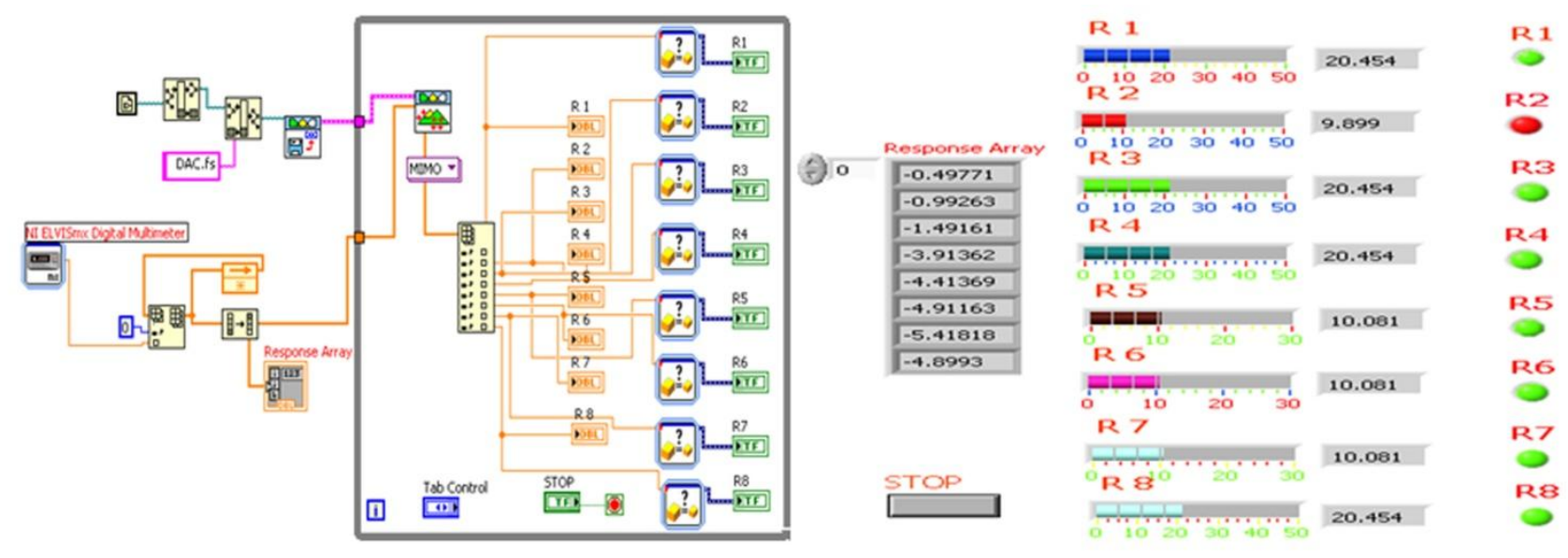

(a)

(b)

Fig.3 Proposed Virtual Instrument for fault diagnosis in real time validation (a) Block diagram (b) Front panel

\section{RESULTS AND DISCUSSION}

In this section results of parametric fault diagnosis using intelligent virtual instrument in $\mathrm{R} 2 \mathrm{R}$ digital to analog converter circuit with simulated and with the real time data are presented. In both the cases fault diagnosis results are checked for the entire defined parametric fault models (R1 $\uparrow$, $\mathrm{R} 1 \downarrow, \mathrm{R} 2 \uparrow, \mathrm{R} 2 \downarrow, \mathrm{R} 3 \uparrow, \mathrm{R} 3 \downarrow, \mathrm{R} 4 \uparrow, \mathrm{R} 4 \downarrow, \mathrm{R} 5 \uparrow, \mathrm{R} 5 \downarrow, \mathrm{R} 6 \uparrow, \mathrm{R} 6 \downarrow$, $\mathrm{R} 7 \uparrow, \mathrm{R} 7 \downarrow, \mathrm{R} 8 \uparrow$ and $\mathrm{R} 8 \downarrow$ ) in the R2R network of DAC. It has been concluded that in both the cases the fault diagnosis efficiency is $100 \%$. Here one of the sample results of fault diagnosis during simulation study is shown in fig.2. A set of output responses $(-0.4886,-0.9843,-1.475,-2.046,-2.5$, $3.002,-3.512,-5.015)$ of the circuit under test obtained from the Monte Carlo run for the fault model R5 $\uparrow$ is applied to the virtual instrument output. In the front panel fault indicator shows that the component R5 is faulty and at the same time the estimated values of the components are also displayed. These are $\mathrm{R} 1=\mathrm{R} 2=\mathrm{R} 3=\mathrm{R} 4=\mathrm{R} 8=19.931 \mathrm{~K} \Omega, \mathrm{R} 6=\mathrm{R} 7=10.133$ $\mathrm{K} \Omega$ and $\mathrm{R} 5=15.399 \mathrm{~K} \Omega$. It is clear from the result that all the resistances except R5 are within their tolerance limits and the value of R5 indicates that fault model R5 $\uparrow$ has been detected.

The results of fault diagnosis for fault model R $2 \downarrow$ in real time validation of the method is shown in fig. 3. A set of output responses $(-0.49771,-0.99263,-1.49161,-3.91362,-4.41369$, $-4.91163,-5.41818,-4.8993)$ are sampled with digital multimeter tool of NI ELVIS II board for fault model R2 $\downarrow$.
The output indicator shows that $\mathrm{R} 2$ resistance is faulty. The estimated values of the components are displayed are $\mathrm{R} 1=\mathrm{R} 3=\mathrm{R} 4=\mathrm{R} 8=20.454 \mathrm{~K} \Omega, \quad \mathrm{R} 5=\mathrm{R} 6=\mathrm{R} 7=10.081 \mathrm{~K} \Omega$ and $\mathrm{R} 2=9.899 \mathrm{~K} \Omega$. The results show that resistance $\mathrm{R} 2$ is faulty and all other components are well within their tolerance limits.

\section{CONCLUSION}

In this paper, a design of intelligent virtual instrument for the diagnosis of parametric faults in $\mathrm{R} 2 \mathrm{R}$ digital to analog converter circuit has been presented. The fuzzy classifier is implemented for fault classification. It not only classifies the parametric single faults but also gives the estimated value of the faulty component with good accuracy. Combination of the fuzzy classifier and the virtual instrument enables the diagnosis result to be visually and directly displayed. The fault diagnosis results are presented with good accuracy for a benchmark R2R digital to analog converter circuit with simulated data obtained with MULTISIM software and also with the real time data obtained with hardware implementation of the circuit on NI ELVIS II board.

\section{ACKNOWLEDGEMENTS}

Authors are greatly indebted to the department of Electronics and Communication Engineering \& Technology, SLIET, Longowal-148106 (District: Sangrur), Punjab, India for providing excellent lab facilities that make this work feasible. 


\section{REFERENCES}

[1] Liu, E. Kao, W., Felt, E. 1994. Analog testability analysis and fault diagnosis using behavioral modeling, Custom Integrated Circuits Conference, pp. 413-416. 1994.

[2] Charles Stroud, Jason Morton, Atia Islam and Hazem Alassaly. 2003. A Mixed-Signal Built-In Self-Test Approach for Analog Circuits, Southwest Symposium on Mixed-Signal Design, pp. 196-201, 2003.

[3] P.Kalpana, K.Gunavathi, 2004. Fault oriented Test Pattern Generator for Digital to Analog converters, Academic Open Internet Journal, Volume 13, 2004.

[4] Ramesh, J. Srinivasulu, M.; Gunavathi, K. 2009. A novel on chip circuit for fault detection in digital to analog converters, International Conference on Control, Automation, Communication and Energy Conservation, pp. 1-8, 2009.

[5] P. Kalpana, K. Gunavathi, 2007. A Novel Implicit Parametric Fault Detection Method for Analog/Mixed Signal Circuits Using Wavelets, ICGST-PDCS Journal, Volume 7, Issue 1, pp. 43-48, May, 2007.
[6] William G. Fenton, T. M. McGinnity, and Liam P.Maguire, 2001. Fault Diagnosis of Electronic Systems Using Intelligent Techniques: A Review, IEEE Transactions on Systems, Man, and Cybernetics - part c: Applications and Reviews 31, No.3 pp. 269-281. 2001.

[7] Peng Wang, Shiyuan Yang, 2005. A New Diagnosis Approach for Handling Tolerence in Analog and MixedSignal Circuits by Using Fuzzy Math, IEEE Transactions on Circuits and Systems-I: Regular Papers, Vol. 52, No.10, pp. 2118-2127, 2005.

[8] B. Kaminaska, K. Arabi, I. Bell, p. Goteti, J.L Huertas, B. Kim, A. Rueda, M.Soma, 1997. Analog and Mixedsignal Benchmark Circuits- First Release, International Test Conference, pp. 183-190, 1997.

[9] Mihai IORDACHE, Lucia DUMITRIU, Dragos NICULAE, 2008. On The Sensitivity Analysis Of Analog Circuits, Annals of the University of Craiova, Electrical Engineering series, No. 32, pp. 11-16, 2008.

[10] Jafferey Travis, Jim Kring, 2009 LabVIEW for Everyone: Graphical Programming Made Easy and Fun, Pearson Education, 2009. 